\title{
NMR shieldings from density functional perturbation theory: GIPAW versus all-electron calculations.
}

\section{Supplementary material}

\author{
G. A. de Wijs, ${ }^{1}$ R. Laskowski, ${ }^{2}$ P. Blaha,${ }^{3}$ R. W. A. Havenith,${ }^{4,5}$ G. Kresse,${ }^{6}$ and M. Marsman ${ }^{6}$ \\ ${ }^{1}$ Radboud University, Institute for Molecules and Materials, \\ Heyendaalseweg 135, NL-6525 AJ Nijmegen, The Netherlands \\ ${ }^{2}$ Institute of High Performance Computing, $A * S T A R$, \\ 1 Fusionopolis Way, \#16-16, Connexis, Singapore 138632 \\ ${ }^{3}$ Institute of Materials Chemistry, Vienna University of Technology, \\ Getreidemarkt 9/165-TC, A-1060 Vienna, Austria \\ ${ }^{4}$ Zernike Institute for Advanced Materials, Stratingh Institute for Chemistry, \\ University of Groningen, Nijenborgh 4, NL-9747 AG Groningen, The Netherlands \\ ${ }^{5}$ Ghent Quantum Chemistry Group, Department of Inorganic and Physical Chemistry, \\ Ghent University, Krijgslaan 281 (S3), B-9000 Gent, Belgium \\ ${ }^{6}$ Faculty of Physics and Center for Computational Materials Science, \\ University of Vienna, Sensengasse 8/12, A-1090 Vienna, Austria
}

Calculated shieldings calculated without the susceptibility contribution are in Table S1. Coordinates of the atoms of the molecules with $\mathrm{Al}, \mathrm{Si}$ and $\mathrm{P}$ nuclei are in Tables \$2, S3 and S4 respectively.

TABLE S1: Isotropic NMR shieldings without $G=0$ contribution (ppm). Other details as in Table III.

\begin{tabular}{|c|c|c|c|}
\hline & \multirow{2}{*}{ WIEN2k } & \multicolumn{2}{|c|}{ VASP } \\
\hline & & optim. & standard \\
\hline \multicolumn{4}{|c|}{ F shieldings } \\
\hline $\mathrm{NaF}$ & 385.04 & 383.14 & 386.38 \\
\hline $\mathrm{LiF}$ & 360.95 & 360.37 & 361.10 \\
\hline $\mathrm{InF}_{3}$ & 353.01 & 353.22 & 356.83 \\
\hline $\mathrm{MgF}_{2}$ & 352.78 & 352.16 & 354.54 \\
\hline$\alpha-\mathrm{AlF}_{3}$ & 325.72 & 325.03 & 326.57 \\
\hline $\mathrm{GaF}_{3}$ & 299.85 & 298.47 & 302.27 \\
\hline $\mathrm{KF}$ & 262.64 & 261.37 & 262.91 \\
\hline $\mathrm{RbF}$ & 213.55 & 213.45 & 215.99 \\
\hline $\mathrm{CaF}_{2}$ & 211.92 & 210.88 & 212.26 \\
\hline $\mathrm{SrF}_{2}$ & 206.32 & 206.43 & 210.28 \\
\hline $\mathrm{TlF}$ & 132.65 & 136.26 & 138.32 \\
\hline CsF & 115.77 & 116.55 & 124.85 \\
\hline $\mathrm{BaF}_{2}$ & 115.56 & 118.34 & 144.00 \\
\hline $\operatorname{MAD}(\mathrm{F})$ & 0.00 & 1.16 & 4.66 \\
\hline \multicolumn{4}{|c|}{ O shieldings } \\
\hline $\mathrm{BeO}$ & 221.48 & 221.24 & 220.56 \\
\hline $\mathrm{SiO}_{2}$ & 205.26 & 204.64 & 205.21 \\
\hline $\mathrm{MgO}$ & 190.04 & 186.67 & 189.09 \\
\hline $\mathrm{BaSnO}_{3}$ & 71.47 & 72.81 & 82.55 \\
\hline $\mathrm{CaO}$ & -151.26 & -152.79 & -153.15 \\
\hline $\mathrm{BaZrO}_{3}$ & -182.14 & -179.20 & -171.87 \\
\hline $\mathrm{SrO}$ & -220.06 & -225.67 & -224.90 \\
\hline $\mathrm{SrTiO}_{3}$ & -292.89 & -291.97 & -297.44 \\
\hline $\mathrm{BaTiO}_{3}$ & -363.75 & -361.88 & -358.88 \\
\hline$D_{0} 1103$ & -369.49 & -367.59 & -363.91 \\
\hline $\mathrm{BaO}$ & -487.13 & -489.80 & -468.58 \\
\hline $\operatorname{MAD}(\mathrm{O})$ & 0.00 & 2.09 & 5.78 \\
\hline
\end{tabular}


TABLE S2: Atomic positions for molecules containing Al nuclei.

\begin{tabular}{|c|c|c|c|c|}
\hline & & $x(\AA)$ & $y(\AA)$ & $z(\AA)$ \\
\hline \multirow[t]{5}{*}{$\overline{\left[\mathrm{AlH}_{4}\right]}$} & $\overline{\mathrm{Al}}$ & 3.499929 & 3.499929 & 3.147370 \\
\hline & $\mathrm{H}$ & 4.434678 & 2.565180 & 2.189269 \\
\hline & $\mathrm{H}$ & 2.565180 & 2.565180 & 4.105461 \\
\hline & $\mathrm{H}$ & 2.565180 & 4.434678 & 2.189269 \\
\hline & $\mathrm{H}$ & 4.434678 & 4.434678 & 4.105461 \\
\hline \multirow[t]{5}{*}[\mathrm{AlH}_{4}]{$^{-}$} & $\mathrm{Al}$ & 3.538681 & 2.270439 & 5.289092 \\
\hline & $\mathrm{H}$ & 2.224501 & 3.237801 & 5.289092 \\
\hline & $\mathrm{H}$ & 4.852860 & 3.237801 & 5.289092 \\
\hline & $\mathrm{H}$ & 3.538681 & 1.287091 & 3.994212 \\
\hline & $\mathrm{H}$ & 3.538681 & 1.287091 & 6.583967 \\
\hline \multirow[t]{5}{*}[\mathrm{AlH}_{4}]{$^{-}$} & $\mathrm{Al}$ & 3.367010 & 3.166802 & 2.160921 \\
\hline & $\mathrm{H}$ & 3.367010 & 3.378023 & .546772 \\
\hline & $\mathrm{H}$ & 2.071760 & 3.912999 & 2.824731 \\
\hline & $\mathrm{H}$ & 4.662261 & 3.912999 & 2.824731 \\
\hline & $\mathrm{H}$ & 0 & & 51 \\
\hline \multirow[t]{5}{*}[\mathrm{AlH}_{4}]{$^{-}$} & $\mathrm{Al}$ & 6.4 & 89 & 3.727782 \\
\hline & $\mathrm{H}$ & 7.537068 & 2.3 & 4.763868 \\
\hline & $\mathrm{H}$ & 5.326061 & 3.596451 & 4.763868 \\
\hline & $\mathrm{H}$ & 7.133835 & 4.109440 & 2.835542 \\
\hline & $\mathrm{H}$ & 5.729294 & 1.812738 & 2.835542 \\
\hline \multirow[t]{8}{*}{$\mathrm{Al}_{2} \mathrm{H}_{6}$} & $\mathrm{Al}$ & 1.290911 & .000000 & .000000 \\
\hline & $\mathrm{Al}$ & -1.290911 & .000000 & .000000 \\
\hline & $\mathrm{H}$ & .000000 & .000000 & 1.160480 \\
\hline & $\mathrm{H}$ & .000000 & .000000 & -1.160480 \\
\hline & $\mathrm{H}$ & 1.971661 & 1.430667 & .000000 \\
\hline & $\mathrm{H}$ & 1.971661 & -1.430667 & .000000 \\
\hline & $\mathrm{H}$ & -1.971661 & 1.430667 & .000000 \\
\hline & $\mathrm{H}$ & -1.971661 & -1.430667 & .000000 \\
\hline \multirow[t]{4}{*}{$\mathrm{AlH}_{3}$} & $\mathrm{Al}$ & .000000 & .000000 & .000000 \\
\hline & $\mathrm{H}$ & .000000 & 1.579020 & .000000 \\
\hline & $\mathrm{H}$ & 1.367472 & -.789511 & .000000 \\
\hline & $\mathrm{H}$ & -1.367472 & -.789511 & .000000 \\
\hline
\end{tabular}


TABLE S3: Atomic positions for molecules containing Si nuclei.

\begin{tabular}{rrrrr}
\hline \hline & & \multicolumn{1}{c}{$x(\AA)$} & \multicolumn{1}{c}{$y(\AA)$} & \multicolumn{1}{c}{$z(\AA)$} \\
\hline $\mathrm{Si}_{2} \mathrm{H}_{6}$ & $\mathrm{Si}$ & .000000 & .000000 & 1.174799 \\
& $\mathrm{Si}$ & .000000 & .000000 & -1.174799 \\
& $\mathrm{H}$ & .000000 & 1.389598 & 1.691101 \\
& $\mathrm{H}$ & -1.203401 & -.694799 & 1.691101 \\
& $\mathrm{H}$ & 1.203401 & -.694799 & 1.691101 \\
& $\mathrm{H}$ & .000000 & -1.389598 & -1.691101 \\
& $\mathrm{H}$ & -1.203401 & .694799 & -1.691101 \\
& $\mathrm{H}$ & 1.203401 & .694799 & -1.691101 \\
$\mathrm{SiH}_{4}$ & $\mathrm{Si}$ & .000000 & .000000 & .000000 \\
& $\mathrm{H}$ & .861738 & -.861738 & .861738 \\
& $\mathrm{H}$ & -.861738 & .861738 & .861738 \\
& $\mathrm{H}$ & -.861738 & -.861738 & -.861738 \\
& $\mathrm{H}$ & .861738 & .861738 & .861738 \\
$\mathrm{Si}_{2} \mathrm{H}_{4}$ & $\mathrm{Si}$ & 1.052200 & .000000 & .217920 \\
& $\mathrm{Si}$ & -1.052200 & .000000 & -.217920 \\
& $\mathrm{H}$ & 1.838784 & 1.244259 & -.026953 \\
& $\mathrm{H}$ & 1.838784 & -1.244259 & -.026953 \\
& $\mathrm{H}$ & -1.838784 & 1.244259 & .026953 \\
& $\mathrm{H}$ & -1.838784 & -1.244259 & .026953 \\
$\mathrm{SiH}_{2}$ & $\mathrm{Si}$ & .000000 & .000000 & .132600 \\
& $\mathrm{H}$ & .000000 & 1.091200 & -.928097 \\
& $\mathrm{H}$ & .000000 & -1.091200 & -.928097 \\
\hline \hline & & & &
\end{tabular}

TABLE S4: Atomic positions for molecules containing $\mathrm{P}$ nuclei.

\begin{tabular}{|c|c|c|c|c|}
\hline & & $x(\check{A})$ & $y(\check{A})$ & $z(\check{A})$ \\
\hline \multirow[t]{4}{*}{$\mathrm{P}_{4}$} & $\mathrm{P}$ & .772319 & .772319 & .772319 \\
\hline & $\mathrm{P}$ & -.772319 & -.772319 & .772319 \\
\hline & $\mathrm{P}$ & -.772319 & .772319 & -.772319 \\
\hline & $\mathrm{P}$ & .772319 & -.772319 & -.772319 \\
\hline \multirow[t]{4}{*}{$\mathrm{PH}_{3}$} & $\mathrm{P}$ & .000000 & .000000 & .000000 \\
\hline & $\mathrm{H}$ & .945009 & .508249 & .945009 \\
\hline & $\mathrm{H}$ & .945009 & -.945009 & -.508249 \\
\hline & $\mathrm{H}$ & -.508249 & -.945009 & .945009 \\
\hline \multirow{6}{*}{$\mathrm{P}_{2} \mathrm{H}_{4}$} & $\mathrm{P}$ & .000000 & 1.119312 & -.085740 \\
\hline & $\mathrm{P}$ & .000000 & -1.119312 & -.085740 \\
\hline & $\mathrm{H}$ & -.191443 & 1.364852 & 1.296710 \\
\hline & $\mathrm{H}$ & 1.409764 & 1.237215 & -.010603 \\
\hline & $\mathrm{H}$ & .191443 & -1.364852 & 1.296710 \\
\hline & $\mathrm{H}$ & -1.409764 & -1.237215 & -.010603 \\
\hline \multirow[t]{8}{*}{$\mathrm{H}_{3} \mathrm{PO}_{4}$} & $\mathrm{P}$ & .000000 & .000000 & .000000 \\
\hline & $\mathrm{O}$ & -.706579 & 1.156170 & -.569987 \\
\hline & $\mathrm{O}$ & -.893018 & -.897538 & 1.007560 \\
\hline & $\mathrm{O}$ & .581529 & -1.121920 & -.991408 \\
\hline & $\mathrm{O}$ & 1.325710 & .392790 & .804032 \\
\hline & $\mathrm{H}$ & -.069470 & -1.407680 & -1.657030 \\
\hline & $\mathrm{H}$ & -1.593160 & -.358900 & 1.418850 \\
\hline & $\mathrm{H}$ & 1.824050 & -.371290 & 1.145860 \\
\hline \multirow[t]{4}{*}{$\mathrm{PF}_{3}$} & $\mathrm{P}$ & .000000 & .000000 & .000000 \\
\hline & $\mathrm{F}$ & .000000 & 1.370150 & -.790302 \\
\hline & $\mathrm{F}$ & 1.187690 & -.686336 & -.787314 \\
\hline & $\mathrm{F}$ & -1.187690 & -.686336 & -.787314 \\
\hline \multirow[t]{2}{*}{$\mathrm{P}_{2}$} & $\mathrm{P}$ & .000000 & .000000 & .000000 \\
\hline & $\mathrm{P}$ & .000000 & .000000 & 1.890458 \\
\hline
\end{tabular}




\title{
NMR shieldings from density functional perturbation theory: GIPAW versus all-electron calculations.
}

\author{
G. A. de Wijs, ${ }^{1}$ R. Laskowski, ${ }^{2}$ P. Blaha, ${ }^{3}$ R. W. A. Havenith, ${ }^{4,5}$ G. Kresse,${ }^{6}$ and M. Marsman ${ }^{6}$ \\ 1) Radboud University, Institute for Molecules and Materials, Heyendaalseweg 135, NL-6525 AJ Nijmegen, \\ The Netherlands \\ ${ }^{2)}$ Institute of High Performance Computing, A*STAR, 1 Fusionopolis Way, \#16-16, Connexis, \\ Singapore 138632 \\ ${ }^{3)}$ Institute of Materials Chemistry, Vienna University of Technology, Getreidemarkt 9/165-TC, A-1060 Vienna, \\ Austria \\ 4) Zernike Institute for Advanced Materials, Stratingh Institute for Chemistry, University of Groningen, Nijenborgh 4, \\ NL-9747 AG Groningen, The Netherlands \\ 5) Ghent Quantum Chemistry Group, Department of Inorganic and Physical Chemistry, Ghent University, \\ Krijgslaan 281 (S3), B-9000 Gent, Belgium \\ ${ }^{6)}$ Faculty of Physics and Center for Computational Materials Science, University of Vienna, Sensengasse 8/12, \\ A-1090 Vienna, Austria
}

(Dated: 14 September 2016)

We present a benchmark of the density functional linear response calculation of NMR shieldings within the Gauge-Including Projector-Augmented-Wave method against all-electron Augmented-Plane-Wave+localorbital and uncontracted Gaussian basis set results for NMR shieldings in molecular and solid state systems. In general, excellent agreement between the aforementioned methods is obtained. Scalar relativistic effects are shown to be quite large for nuclei in molecules in the deshielded limit. The small component makes up a substantial part of the relativistic corrections.

\section{INTRODUCTION}

Nuclear magnetic resonance (NMR) spectroscopy presents a powerful and sensitive probe of the structure of molecules, liquids, and solids on the atomic scale. In general, however, the retrieval of structural information from measured NMR spectra is a far from trivial process, since as yet, empirical rules that map between the NMR spectrum and the structure were found to exist only for relatively simple organic molecules. To connect the features of measured NMR spectra unambiguously to complex structural properties therefore remains difficult (and is often impossible) without additional input from $a b$ initio quantum mechanical modeling.

In the case of molecular systems and finite clusters of atoms, the $a b$ initio techniques traditionally used in quantum chemistry have been successfully applied to aid in the analysis of experimental solution-state NMR spectra for quite some time now $\stackrel{1}{\underline{1}}$ In the case of solid-state NMR, finite clusters of atoms were used to approximate the infinite solid. Ab initio quantum mechanical calculations of NMR shieldings in truly extended systems under periodic boundary conditions were first performed by Mauri, Pfrommer, and Louie,$\frac{2}{\underline{\underline{T}}}$ using a linear response approach.

Although Mauri et al. derived their expressions starting from an all-electron Hamiltonian, practical implementations thereof used norm-conserving pseudopotentials, which largely limited its applicability to the calculation of chemical shifts for light elements. Only with the introduction of the Gauge-Including Projector Augmented Wave (GIPAW) method by Pickard and Mauri,,$\frac{3}{}$ and its extension to non-norm-conserving pseudo orbitals by Yates, Pickard, and Mauri (YPM $)^{4}$ several years later, did the calculation of NMR shieldings become routinely possible for most of the nuclei commonly studied in NMR.

The GIPAW method permits to obtain accurate chemical shielding with a plane-wave basis set. As in the original projector-augmented-wave method of Blöchl,,$\frac{5}{5}$ it recovers the shape of the all-electron Kohn-Sham orbitals near the nucleus through an augmentation procedure involving atom-centered functions. In addition, the GIPAW method solves the gauge problem arising from incompleteness of the atom-centered augmentation functions in a way similar as done for molecules in the Gauge Independent Atomic Orbital (GIAO) method. $\underline{6}$ The GIPAW formalism of YPM has been implemented in several plane-wave codes (for instance, CASTEP, 7 Quantum Espresso,,$\frac{8}{-}$ and PARATEC) and is currently widely used in the solid-state NMR community for an extensive range of applications (see, e.g., Ref. 9 and references therein).

Recently the calculation of NMR shieldings within the augmented-plane-wave + local-orbital (APW+lo) method was implemented in WIEN2k, ${ }^{10}$-12 In this paper we benchmark the recent implementation (by several of us) of the linear response calculation of NMR shieldings within the GIPAW formalism of YPM in the Vienna $A b$ initio Simulation Package (VASP) ${ }^{13}$ against all-electron APW+lo results for NMR shieldings in molecular and solid state systems and against non-relativistic LCAO calculations using DALTON (Ref. 14) and large uncontracted Gaussian basis sets. These benchmarks serve to further validate the aforementioned implementation in VASP as well as WIEN2k, and as importantly, to establish the quality of the GIPAW approach and the parameterization of the atomic scattering properties involved.

The rest of this paper is organized as follows: in sec- 
tion $\amalg$ we reiterate the linear response expressions for the NMR shieldings in the GIPAW formalism. The particulars of the PAW data sets we use in our GIPAW NMR benchmarks and the general setup of these calculations are discussed in Sec. III. The results of aforementioned GIPAW benchmarks are presented in Sec. IV] and compared to all-electron calculations and-partly - to experiment. Conclusions are drawn in Sec. V.

\section{THEORY}

The magnetic shielding tensor $\sigma(\mathbf{R})$ at nuclear position $\mathbf{R}$ is found from the ratio of the induced magnetic field at the aforementioned position to an externally applied magnetic field $\mathbf{B}$ :

$$
\sigma_{\alpha \beta}(\mathbf{R})=-\frac{\partial B_{\alpha}^{\mathrm{ind}}(\mathbf{R})}{\partial B_{\beta}} .
$$

The induced magnetic field $\mathbf{B}_{\text {ind }}$ is given by the BiotSavart law,

$$
\mathbf{B}_{\text {ind }}(\mathbf{R})=\frac{1}{c} \int \mathbf{j}_{\text {ind }}(\mathbf{r}) \times \frac{\mathbf{R}-\mathbf{r}}{|\mathbf{R}-\mathbf{r}|^{3}} d \mathbf{r},
$$

where $\mathbf{j}_{\text {ind }}$ is the current induced by the external magnetic field B. The induced current is commonly calculated from the linear response of the system to the external magnetic field.

To first-order in the external magnetic field, $\mathbf{j}_{\text {ind }}$ is given by

$$
\begin{aligned}
\mathbf{j}_{\text {ind }}(\mathbf{r})=\sum_{i \in \text { occ }} & \left(\left\langle\psi_{i}^{(1)}\left|\mathbf{J}^{p}(\mathbf{r})\right| \psi_{i}^{(0)}\right\rangle+\left\langle\psi_{i}^{(0)}\left|\mathbf{J}^{p}(\mathbf{r})\right| \psi_{i}^{(1)}\right\rangle\right. \\
& \left.+\left\langle\psi_{i}^{(0)}\left|\mathbf{J}^{d}(\mathbf{r})\right| \psi_{i}^{(0)}\right\rangle\right),
\end{aligned}
$$

where

$$
\mathbf{J}^{p}(\mathbf{r})=-\frac{\mathbf{p}|\mathbf{r}\rangle\langle\mathbf{r}|+| \mathbf{r}\rangle\langle\mathbf{r}| \mathbf{p}}{2}
$$

and

$$
\mathbf{J}^{d}(\mathbf{r})=-\frac{\mathbf{B} \times \mathbf{r}}{2 c}|\mathbf{r}\rangle\langle\mathbf{r}|
$$

are the paramagnetic and diamagnetic current operators, respectively. In Eq. (3), $\psi^{(0)}$ denote ground state orbitals, i.e., the solutions to

$$
H^{(0)}\left|\psi_{i}^{(0)}\right\rangle=\epsilon_{i}^{(0)}\left|\psi_{i}^{(0)}\right\rangle
$$

and $\psi^{(1)}$ the first-order response of the orbitals to the external magnetic field. The sum in Eq. (3) goes over all occupied states.

In the symmetric gauge, the perturbation of the Hamiltonian to first-order in the external magnetic field, is given by

$$
H^{(1)}=\frac{1}{2 c} \mathbf{L} \cdot \mathbf{B} \text {. }
$$

With the above definition the first-order change in the orbitals is straightforwardly found to be

$$
\left|\psi_{i}^{(1)}\right\rangle=\mathcal{G}\left(\epsilon_{i}^{(0)}\right) H^{(1)}\left|\psi_{i}^{(0)}\right\rangle
$$

where $\mathcal{G}$ is the Green's function

$$
\mathcal{G}(\epsilon)=\sum_{j \in \operatorname{vir}} \frac{\left|\psi_{j}^{(0)}\right\rangle\left\langle\psi_{j}^{(0)}\right|}{\epsilon-\epsilon_{j}^{(0)}}
$$

and the sum is over all empty (virtual) orbitals. Commonly, the sum over empty orbitals is avoided by recasting Eq. (8) as a Sternheimer equation:

$$
\left(\epsilon_{i}^{(0)}-H^{(0)}\right)\left|\psi_{i}^{(1)}\right\rangle=P_{c} H^{(1)}\left|\psi_{i}^{(0)}\right\rangle
$$

to be solved for $\psi_{i}^{(1)}$. In the above $P_{c}=1-$ $\sum_{i \in \text { occ }}\left|\psi_{i}^{(0)}\right\rangle\left\langle\psi_{i}^{(0)}\right|$ represents a projection onto the virtual subspace.

In plane wave based implementations, Eq. (2) is most conveniently evaluated in reciprocal space,

$$
\mathbf{B}_{\text {ind }}(\mathbf{R})=\frac{4 \pi i}{c} \sum_{\mathbf{G} \neq 0} \frac{\mathbf{G} \times \mathbf{j}_{\text {ind }}(\mathbf{G})}{G^{2}} e^{i \mathbf{G} \cdot \mathbf{R}},
$$

where $\mathbf{G}$ are the reciprocal space vectors. In solid state systems there is an additional contribution at $\mathbf{G}=0$, i.e., a uniform field, that is determined by the shape of the sample and the macroscopic magnetic susceptibility tensor $\chi$. For a spherical sample this contribution is given by:

$$
\mathbf{B}_{\text {ind }}(\mathbf{G}=0)=\frac{8 \pi}{3} \stackrel{\leftrightarrow}{\chi} \mathbf{B}
$$

The magnetic susceptibility tensor may be numerically calculated as proposed by Mauri and Louie, 15

$$
\overleftrightarrow{\chi}=\lim _{q \rightarrow 0} \frac{\overleftrightarrow{F}(q)-2 \overleftrightarrow{F}(0)+\overleftrightarrow{F}(-q)}{q^{2}}
$$

where $F_{\alpha \beta}(q)=\left(2-\delta_{\alpha \beta}\right) Q_{\alpha \beta}(q), \alpha, \beta=x, y, z$ are the cartesian directions, and the tensor $Q(q)$ can be written as

$$
\begin{aligned}
& \stackrel{\leftrightarrow}{Q}(q)= \\
& \frac{1}{N_{\mathbf{k}} \Omega c^{2}} \sum_{n \mathbf{k} \in \text { occ }} \sum_{\gamma}^{x, y, z} \operatorname{Re}\left\{\left\langle u_{n \mathbf{k}}^{(0)}\left|\mathbf{A}_{\gamma} \mathcal{G}_{\mathbf{k}+q \hat{\gamma}}\left(\epsilon_{n \mathbf{k}}^{(0)}\right) \mathbf{A}_{\gamma}\right| u_{n \mathbf{k}}^{(0)}\right\rangle\right\}
\end{aligned}
$$

with

$$
\mathbf{A}_{\gamma}=\hat{\mathbf{u}}_{\gamma} \times(\mathbf{p}+\mathbf{k})
$$

In Eq. (14), the functions $u_{n \mathbf{k}}^{(0)}$ denote the cell periodic part of the ground state Bloch orbitals, $N_{\mathbf{k}}$ is the number of $k$-points chosen to sample the first Brillouin zone, 
$\Omega$ the volume of the unit cell, and the sum over $n$ and $\mathbf{k}$ includes all occupied Bloch orbitals. The Green's function in Eq. (14) is given by

$$
\mathcal{G}_{\mathbf{k}}(\epsilon)=\sum_{n \in \text { vir }} \frac{\left|u_{n \mathbf{k}}^{(0)}\right\rangle\left\langle u_{n \mathbf{k}}^{(0)}\right|}{\epsilon-\epsilon_{n \mathbf{k}}^{(0)}} .
$$

In practice, we have implemented Eqs. (1)-(16) within the Gauge-Including Projector-Augmented-Wave (GIPAW) method of Yates, Pickard, and Mauri (YPM) $\underline{\underline{4}}$ The GIPAW deals with several numerical issues that plague the PAW method (in a uniform magnetic field): (a) it reestablishes the translational symmetry that is

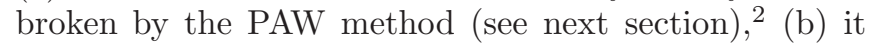
balances the different rates of convergence of the paraand diamagnetic contributions to the induced current which also affects translational symmetry - via the generalized $f$-sum rule,,$\stackrel{2}{=}$ and (c) it solves the position operator problem with the help of a reciprocal space modulation vector q (cf. Eq. [13).

\section{A. GIPAW}

In the Projector-Augmented-Wave (PAW) method of Blöchl the one-electron orbitals $\psi_{n}$ are derived from pseudo (PS) orbitals $\widetilde{\psi}_{n}$ by means of a linear transformation $^{5}$

$$
\left|\psi_{n}\right\rangle=\mathcal{T}\left|\widetilde{\psi}_{n}\right\rangle
$$

with

$$
\mathcal{T}=1+\sum_{j}\left(\left|\phi_{j}\right\rangle-\left|\tilde{\phi}_{j}\right\rangle\right)\left\langle\tilde{p}_{j}\right|
$$

The PS orbitals $\widetilde{\psi}_{n}$ are the variational quantities of the PAW method and are expanded in plane waves. The additional local basis functions, $\phi_{j}$ and $\widetilde{\phi}_{j}$, are nonzero only within non-overlapping spheres centered at the atomic sites $\mathbf{R}_{j}$, the so-called PAW spheres. In the interstitial region between the PAW spheres, therefore, the true one-electron orbitals $\psi_{n}$ are identical to the PS orbitals $\widetilde{\psi}_{n}$. Inside the spheres the PS orbitals are only a computational tool and a bad approximation to the true orbitals, since not even the norm of the true orbital is reproduced. In all practical implementations of the PAW method, the all-electron (AE) partial-waves $\phi_{j}$ are chosen to be solutions of the spherical (scalar relativistic) Schrödinger equation for a non-spinpolarized atom at a specific energy $\varepsilon_{j}$, and for a specific angular momentum $l_{j}$. The pseudo partial waves $\widetilde{\phi}_{j}$ are equivalent to their AE counterparts outside a core radius $r_{c}$ and match continuously onto $\phi_{j}$ inside this radius. In the PAW data sets distributed with VASP they are constructed in accordance with a revised Rappe, Rabe, Kaxiras, and Joannopoulos (RRKJ) scheme, 16,17 The projector functions $\widetilde{p}_{j}$ are constructed to be dual to the PS partial waves, i.e.,

$$
\left\langle\widetilde{p}_{j} \mid \widetilde{\phi}_{j^{\prime}}\right\rangle=\delta_{j j^{\prime}}
$$

A detailed construction recipe for the projector functions can be found in Ref. 17. For a comprehensive introduction to the PAW method we refer the reader to the seminal paper of Blöchl (Ref. 5) and the work of Kresse and Joubert 18

In a uniform magnetic field $\mathbf{B}$ there is an additional complication as the ground state orbitals acquire an additional phase factor upon translation over a vector $\mathbf{t}$, in accordance with:

$$
\left\langle\mathbf{r} \mid \psi_{n}^{\mathbf{t}}\right\rangle=e^{\frac{i}{2 c} \mathbf{r} \cdot \mathbf{t} \times \mathbf{B}}\left\langle\mathbf{r}-\mathbf{t} \mid \psi_{n}\right\rangle
$$

(in the symmetric gauge). This additional phase factor causes a very slow convergence of the linear transformation of Eq. 17 with respect to the number of projectors $\tilde{p}_{j}$. To solve this problem, Pickard and Mauri introduced the so-called Gauge-Including PAW transformation that includes the aforementioned phase factor explicitly: $: 3,4$

$$
\overline{\mathcal{T}}=1+\sum_{j} e^{\frac{i}{2 c} \mathbf{r} \cdot \mathbf{R}_{j} \times \mathbf{B}}\left(\left|\phi_{j}\right\rangle-\left|\tilde{\phi}_{j}\right\rangle\right)\left\langle\tilde{p}_{j}\right| e^{-\frac{i}{2 c} \mathbf{r} \cdot \mathbf{R}_{j} \times \mathbf{B}} .
$$

Using the transformation of Eq. 21, it is straightforward to show that with any local operator $O$ acting on $\psi_{n}$ the GIPAW associates a PS operator $\bar{O}$ acting on the PS orbitals $\bar{\psi}_{n}$ :

$$
\bar{O}=O+\sum_{j j^{\prime}}\left|\bar{p}_{j}\right\rangle\left(\left\langle\bar{\phi}_{j}|O| \bar{\phi}_{j^{\prime}}\right\rangle-\left\langle\overline{\tilde{\phi}}_{j}|O| \overline{\tilde{\phi}}_{j^{\prime}}\right\rangle\right)\left\langle\bar{p}_{j^{\prime}}\right|
$$

where

$$
\begin{aligned}
\left|\bar{p}_{j}\right\rangle & =e^{\frac{i}{2 c} \mathbf{r} \cdot \mathbf{R}_{j} \times \mathbf{B}}\left|\tilde{p}_{j}\right\rangle, \\
\left|\bar{\phi}_{j}\right\rangle & =e^{\frac{i}{2 c} \mathbf{r} \cdot \mathbf{R}_{j} \times \mathbf{B}}\left|\phi_{j}\right\rangle, \\
\left|\tilde{\phi}_{j}\right\rangle & =e^{\frac{i}{2 c} \mathbf{r} \cdot \mathbf{R}_{j} \times \mathbf{B}}\left|\tilde{\phi}_{j}\right\rangle .
\end{aligned}
$$

As shown by YPM, to first-order in the magnetic field B, the GIPAW transformation of the induced current is given by

$$
\begin{aligned}
\mathbf{j}_{\text {ind }}^{(1)}(\mathbf{r})=\sum_{i}^{\text {occ }} & \left(2 \operatorname{Re}\left\{\left\langle\bar{\psi}_{i}^{(0)}\left|\overline{\mathbf{J}}^{(0)}(\mathbf{r})\right| \bar{\psi}_{i}^{(1)}\right\rangle\right\}\right. \\
& -\sum_{j}^{\text {occ }}\left\langle\bar{\psi}_{i}^{(0)}\left|\overline{\mathbf{J}}^{(0)}(\mathbf{r})\right| \bar{\psi}_{j}^{(0)}\right\rangle\left\langle\bar{\psi}_{j}^{(0)}\left|\bar{S}^{(1)}\right| \bar{\psi}_{i}^{(0)}\right\rangle \\
& \left.+\left\langle\bar{\psi}_{i}^{(0)}\left|\overline{\mathbf{J}}^{(1)}(\mathbf{r})\right| \bar{\psi}_{i}^{(0)}\right\rangle\right)
\end{aligned}
$$

In the above, $\bar{\psi}^{(0)}$ are the ground state orbitals, i.e., the solutions to

$$
\bar{H}^{(0)}\left|\bar{\psi}_{n}^{(0)}\right\rangle=\epsilon_{n}^{(0)} \bar{S}^{(0)}\left|\bar{\psi}_{n}^{(0)}\right\rangle .
$$

This equation is the GIPAW transform of the KohnSham equations (see Eq. 6) to zeroth-order in the magnetic field, so it is just the usual PAW generalized KohnSham eigenvalue equation (i.e., $\bar{H}^{(0)}$ and $\bar{S}^{(0)}$ are equal 
to the PAW Hamiltonian and overlap operators of Refs. 5 and 18 , and consequently $\bar{\psi}_{n}^{(0)}=\tilde{\psi}_{n}$ and $\left.\epsilon_{n}^{(0)}=\epsilon_{n}\right)$.

The current operators, to zeroth- and first-order in the magnetic field, are given by

$$
\overline{\mathbf{J}}^{(0)}=\mathbf{J}^{p}(\mathbf{r})+\sum_{\mathbf{R}} \Delta \mathbf{J}_{\mathbf{R}}^{p}(\mathbf{r})
$$

and

$\overline{\mathbf{J}}^{(1)}=\mathbf{J}^{d}(\mathbf{r})+\sum_{\mathbf{R}}\left(\Delta \mathbf{J}_{\mathbf{R}}^{d}(\mathbf{r})+\frac{1}{2 c i}\left[\mathbf{B} \times \mathbf{R} \cdot \mathbf{r}, \Delta \mathbf{J}_{\mathbf{R}}^{p}(\mathbf{r})\right]\right)$,

respectively. These are easily recognized as the paramagnetic and diamagnetic current operators of Eqs. 4] and 5, plus additional one-center correction terms (the terms involving $\Delta \mathbf{J}_{\mathbf{R}}^{p}$ and $\Delta \mathbf{J}_{\mathbf{R}}^{d}$; see Ref. 4 ).

For non-normconserving PAW data sets the induced current of Eq. (24) contains an additional contribution connected to the first-order change of the GIPAW orbital overlap operator with respect to the magnetic field,

$$
\bar{S}^{(1)}=\frac{1}{2 c} \sum_{\mathbf{R}} \mathbf{R} \times \frac{1}{i}\left[\mathbf{r}, Q_{\mathbf{R}}\right] \cdot \mathbf{B} .
$$

The first-order change in the GIPAW wave functions $\bar{\psi}^{(1)}$, is found by solving a generalized Sternheimer equation,

$$
\left(\epsilon_{i}^{(0)} \bar{S}^{(0)}-\bar{H}^{(0)}\right)\left|\bar{\psi}_{i}^{(1)}\right\rangle=P_{c}\left(\bar{H}^{(1)}-\epsilon_{i}^{(0)} \bar{S}^{(1)}\right)\left|\bar{\psi}_{i}^{(0)}\right\rangle,
$$

where

$$
P_{c}=1-\sum_{i \in \mathrm{occ}} \bar{S}^{(0)}\left|\bar{\psi}_{i}^{(0)}\right\rangle\left\langle\bar{\psi}_{i}^{(0)}\right|
$$

and

$$
\bar{H}^{(1)}=\frac{1}{2 c}\left(\mathbf{L}+\sum_{\mathbf{R}} \mathbf{R} \times \frac{1}{i}\left[\mathbf{r}, V_{\mathbf{R}}^{\mathrm{nl}}\right]+\sum_{\mathbf{R}} \mathbf{L}_{\mathbf{R}} Q_{\mathbf{R}}\right) \cdot \mathbf{B},
$$

is the first-order contribution to the GIPAW Hamiltonian.

The macroscopic magnetic susceptibility is calculated in accordance with the ansatz of YPM [see Eqs. (47) and (48) of Ref. 4], which equals Eqs. (13)-(16) for $\mathcal{T}=1$, but represents an approximation otherwise. We will not repeat the expressions here.

\section{B. Core contributions}

As was shown by Gregor, Mauri, and Car ${ }^{19}$ the contribution of the core electrons to the NMR shieldings is essentially rigid, and can be calculated from the atomic orbitals of the core electrons:

$$
\sigma_{\alpha \beta}^{c}(\mathbf{R})=\frac{1}{2 c} \sum_{i}^{\text {core }}\left\langle\psi_{i}\left|\frac{1}{r}\right| \psi_{i}\right\rangle \delta_{\alpha \beta},
$$

where the sum is understood to be taken over the core electronic states at atomic site $\mathbf{R}$, and the delta function expresses the fact that the core electrons only contribute isotropically. Of course, in the PAW formalism, we use frozen core states.

As is usual in the GIPAW, the excitations from the valence to the core states are not included in the Green's function (the pseudo equivalent of Eq. 16). In principle these should be included in the proper decoupling of the valence and core contributions to the chemical shieldings, however, in IGAIM (Individual Gauges for Atoms In Molecules) and similar methods their neglect gives rise to errors much smaller than one ppm. ${ }^{19}$ Moreover, such inaccuracies, should they play a role, can be minimized by unfreezing the shallowest core shell(s).

The contribution of the core electrons to the macroscopic magnetic susceptibility is only approximately rigid ${ }^{15}$ It is commonly assumed to be rigid, though, and included as:

$$
\chi_{\alpha \beta}^{c}=-\frac{1}{\Omega c^{2}} \sum_{i}^{\text {core }}\left\langle\psi_{i}\left|r^{2}\right| \psi_{i}\right\rangle \delta_{\alpha \beta},
$$

where the sum is now taken over all core electronic states of the system.

\section{COMPUTATIONAL SETUP}

All calculations employed the Perdew, Burke and Ernzerhof generalized gradient expansion, 20,21

\section{A. GIPAW}

We carried out two series of GIPAW calculations: one with the standard data sets that are designed for general use, ${ }^{22}$ and one where we selected data sets to aim for high accuracy of the shieldings. The former, referred to as "standard" or "stand" below, are a compromise between many demands and should yield good performance for a reasonable plane wave cutoff energy. They are not expected to be optimal for calculating shieldings. Accurate shieldings require an accurate $\mathrm{PAW}$ reconstruction of states in the immediate vicinity of the nucleus, as currents in this region have a high impact on the field at the nucleus. Such accuracy is only needed in the calculation of few properties, that are typically related to spectroscopic techniques probing the nucleus, e.g. for electric field gradients and NMR. The latter series, referred to as "optimal", is intended to give high accuracy for the shieldings. Details of the data sets are compiled in Table Completeness in the projectors and partial waves is easier to realize when the pseudo partial waves are normconserving and have an extra (radial) node for each additional projector (within the same angular momentum channel). Logically this results in harder projector functions. This is the case for the *_sv_GW_nc data sets (see 
Ref. 23). Typically these also have a substantial part of the core states unfrozen, which helps in keeping the PAW matching radii small. Alternatively, for some elements we used just very hard data sets $\left(* \_h\right)$. For the "standard" and "optimal" series we used kinetic energy cutoffs of 700 and $900 \mathrm{eV}$ respectively.

Inside the muffin tin spheres, WIEN2k uses a basis that consists of solutions to the scalar-relativistic Schrödinger equation for the spherical atom: these atomic orbitals have a so-called "large" $(A)$ and "small" $(B)$ component. The AE-partial waves of the VASP PAW data sets are solutions to the same scalar-relativistic equation. However, in the VASP PAW data sets the "small"-component $B$ is not retained. Instead the large component $A$ is rescaled to have the correct norm $(\sqrt{\langle A \mid A\rangle+\langle B \mid B\rangle})$. Since the $B$-component becomes appreciable close to the nuclei, such a treatment causes non-negligible errors in the NMR shielding. This turns out to be problematic for some of the molecular systems.

To solve this issue, we recompute the $B$-component on-the-fly, but use it only to evaluate the $\mathrm{AE}$ one-centre contributions to the shielding: the $B$ AE-partial wave is reconstructed as the radial derivative of the $A$ AE-partial wave, rescaled with a ZORA-like expression for the relativistic mass $\stackrel{24}{2}$ Equation. 22 is modified and becomes:

$$
\begin{aligned}
& \bar{O}=O+ \\
& \sum_{j j^{\prime}}\left|\bar{p}_{j}\right\rangle\left(\left\langle\bar{\phi}_{j}^{A}|O| \bar{\phi}_{j^{\prime}}^{A}\right\rangle+\left\langle\bar{\phi}_{j}^{B}|O| \bar{\phi}_{j^{\prime}}^{B}\right\rangle-\left\langle\overline{\tilde{\phi}}_{j}|O| \overline{\tilde{\phi}}_{j^{\prime}}\right\rangle\right)\left\langle\bar{p}_{j^{\prime}}\right|
\end{aligned}
$$

The $A$ and $B$ AE-partial waves are renormalized such that the electron count in each channel inside the sphere is unaffected.

Note that the aforementioned issue only applies to the contributions to the chemical shielding stemming from the valence electronic states. The core contributions to the chemical shieldings always include the contributions of the "small" component explicitly in both, VASP as well as WIEN2k.

\section{B. APW}

The WIEN2k calculations performed in this work apply the formalism described in Refs. 10 and 12. The standard APW basis set is extended with eight additional local orbitals (NMR-LOs) at higher expansion energies for all "chemical" $l+1$ angular momenta using a procedure described in Ref. 10. The Greens function used to represent the perturbation of the ground state is augmented with additional $r \frac{\partial}{\partial r} u$ terms in order to accelerate convergence with respect to the number of NMRLOs $\underline{12}$ The separation of the valence and core states substantially affects the absolute values of the shielding,$\frac{12}{2}$ thus in the current work we apply the corresponding core-correction. All molecular calculations are done with only the 1s state as core for all atoms (except $\mathrm{H}$ ) because of the short

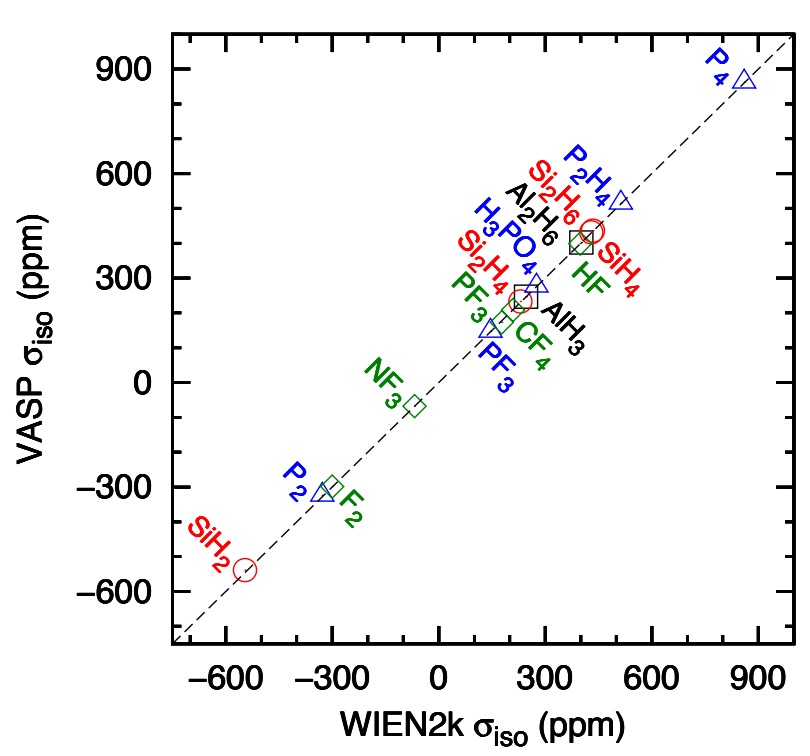

FIG. 1. (Color online) VASP versus WIEN2k PBE Al (black squares), Si (red cicles), P (blue triangles), and F (green diamonds) NMR shieldings. Both VASP (with "optimal" PAW data sets) and WIEN2k results are scalar relativistic with $A$ and $B$ components, i.e. ${ }^{+} B$.

bond-lengths in these molecules, while for the bulk calculations we applied the usual WIEN2k criterion defining valence states as states with atomic eigenvalues above $-6 \mathrm{Ry}$. Core-states are treated fully-relativistically, but in the self-consistent spherical potential only and the corresponding NMR-shielding is calculated via Eq. 32. The numerical parameters are set to standard WIEN2k values. The convergence with respect to the basis set size (RKMAX) has been tested and the presented values of the shielding for the molecular systems (in Table III) are extrapolated to infinite RKMAX (typically extrapolation shifts the shielding by 1-3 ppm compared to the largest applied RKMAX).

\section{GIAO}

For molecules calculations were carried out with the quantum chemical code DALTON (Refs. 14 and 25) using uncontracted aug-cc-pCVXX (XX=DZ, TZ, QZ, 5Z) basis sets (unless stated otherwise) $\underline{26}-\underline{35}$ Uncontracting the basis sets is crucial to observe a convergence of the shieldings (and a lowering of the total energy) with increasing basis set quality. These calculations were nonrelativistic. 
TABLE I. Frozen core configurations and matching core radii $r_{c}$ for $s, p, d$ and $d$ partial waves in atomic units for the optimal PAW data sets. The number of projectors for each quantum number $\ell$ is given in parenthesis. An " $l$ " indicates this is taken as local potential.

\begin{tabular}{|c|c|c|c|c|c|c|}
\hline & frozen core & $s$ & & $\bar{p}$ & $d$ & $\overline{\bar{f}}$ \\
\hline$\overline{\mathrm{H}}$ & & $1.10(2)$ & & $1.10(1)$ & & \\
\hline Li_sv & & $1.40(1)$ & $1.70(1)$ & $1.40(1)$ & $1.40(1) l$ & \\
\hline Be_sv & & $1.50(3)$ & & $1.80(2)$ & $1.80(1) l$ & \\
\hline C_h & {$[\mathrm{He}]$} & $1.10(2)$ & & $1.10(2)$ & $1.10(1) l$ & \\
\hline N_h & {$[\mathrm{He}]$} & $1.10(2)$ & & $1.10(2)$ & $1.10(1) l$ & \\
\hline $\mathrm{O} \_\mathrm{h}$ & {$[\mathrm{He}]$} & $1.10(2)$ & & $1.10(2)$ & $1.10(1) l$ & \\
\hline F_h & {$[\mathrm{He}]$} & $0.85(2)$ & & $1.10(2)$ & $1.10(1) l$ & \\
\hline Na_sv_GW_nc & {$[\mathrm{He}]$} & $1.20(2)$ & & $2.20(3)$ & $2.20(2)$ & \\
\hline Mg_sv_GW_nc & {$[\mathrm{He}]$} & $1.15(1)$ & $1.30(2)$ & $1.65(3)$ & $1.65(2)$ & \\
\hline Al_sv_GW_nc & {$[\mathrm{He}]$} & $1.75(3)$ & & $2.00(3)$ & $1.80(2)$ & $2.00(1)$ \\
\hline Si_sv_GW_nc & {$[\mathrm{He}]$} & $1.70(3)$ & & $1.95(3)$ & $1.70(2)$ & $2.00(1)$ \\
\hline Si_sv_GW_nc & [He] & $1.70(3)$ & & $1.95(3)$ & $1.70(2)$ & $2.00(1)$ \\
\hline P_sv_GW_nc & {$[\mathrm{He}]$} & $1.70(3)$ & & $1.95(3)$ & $1.70(2)$ & $2.00(1)$ \\
\hline Cl_GW_nc & {$[\mathrm{Ne}]$} & $1.14(2)$ & & $1.25(2)$ & $1.70(2)$ & $1.70(1) l$ \\
\hline K_sv_GW_nc & {$[\mathrm{Ne}]$} & $0.95(2)$ & & $1.76(3)$ & $2.10(2)$ & $2.10(2)$ \\
\hline Ca_sv_GW_nc & {$[\mathrm{Ne}]$} & $0.90(2)$ & & $1.65(3)$ & $1.90(3)$ & $2.10(1)$ \\
\hline Ti_sv_GW_nc & {$[\mathrm{Ne}]$} & $0.85(2)$ & & $1.41(2)$ & $1.90(3)$ & $1.90(2)$ \\
\hline Ga_sv_GW_nc & {$[\mathrm{Ne}]$} & $1.23(2)$ & $1.55(1)$ & $1.70(3)$ & $1.90(2)$ & $1.90(2)$ \\
\hline Rb_sv_GW_nc & {$[\operatorname{Ar}](3 d)^{10}$} & $1.16(2)$ & & $2.10(3)$ & $2.30(3)$ & $2.10(2)$ \\
\hline Sr_sv_GW_nc & {$[\mathrm{Ar}](3 d)^{10}$} & $1.10(2)$ & & $2.00(3)$ & $2.30(3)$ & $2.10(2)$ \\
\hline Zr_sv_GW_nc & {$[\mathrm{Ar}](3 d)^{10}$} & $1.01(2)$ & & $1.90(3)$ & $2.10(3)$ & $1.90(2)$ \\
\hline In_sv_GW_nc & {$[\mathrm{Ar}](3 d)^{10}$} & $1.66(2)$ & $1.80(1)$ & $2.00(3)$ & $2.20(3)$ & $1.90(2)$ \\
\hline Sn_sv_GW_nc & {$[\mathrm{Ar}](3 d)^{10}$} & $1.60(2)$ & $1.70(1)$ & $2.00(3)$ & $2.20(3)$ & $1.90(2)$ \\
\hline Cs_sv_GW_nc & {$[\mathrm{Ar}](3 d)^{10}(4 p)^{6}(4 d)^{10}$} & $1.30(1)$ & $1.40(2)$ & $2.25(3)$ & $2.60(3)$ & $2.10(2)$ \\
\hline Ba_sv_GW_nc & {$[\mathrm{Ar}](3 d)^{10}(4 p)^{6}(4 d)^{10}$} & $1.30(1)$ & $1.40(2)$ & $2.20(3)$ & $2.50(3)$ & $2.10(2)$ \\
\hline Tl_sv_GW & {$[\mathrm{Kr}](4 d)^{10}$} & $1.75(3)$ & & $1.90(3)$ & $2.15(3)$ & $2.30(2)$ \\
\hline
\end{tabular}

\section{RESULTS}

\section{A. Molecules}

Table II compares chemical shieldings from VASP calculations (with "standard" and with "optimal" PAW potentials) with all-electron WIEN2k results and DALTON all-electron quantum chemical calculations. Here the VASP calculations were carried out in large boxes $\left(16 \times 16 \times 16\right.$ to $\left.17 \times 17 \times 17 \AA^{3}\right)$, in order to remove artificial fields from currents induced in the periodic images and thus allow for comparison with the vacuum results from DALTON. The WIEN2k results used boxes of $16 \times 16 \times 16 \AA^{3}$ only.

To avoid possible confusion with respect to differences in implementation of relativistic effects, we excluded these in the comparison to DALTON results, i.e., all DALTON results are non-relativistic, and we have redone the VASP calculations with PAW data sets generated from non-relativistic atomic calculations. The nonrelativistic results are labelled with NR in Table II

TableIlists scalar-relativistic VASP and WIEN2k calculations of the chemical shielding, as well. As mentioned at the end of Sec. IIIA, VASP calculations normally do not explicitly take contributions from the "small" component into account (the ninth and tenth columns in Table II), whereas WIEN2k does. To elucidate the ef- fect of this approximation we have reconstructed the $B$ component of the scalar-relativistic atomic orbitals in VASP as well, and included their contribution to the chemical shieldings in the column labelled ${ }^{+} B$.

In Fig. 1 we compare WIEN2k and VASP scalarrelativistic shieldings $\left({ }^{+} B\right)$ for all compounds. Overall the correlation is nearly perfect. Below we discuss the differences in detail with the help of Table II

We start with the seemingly unambiguous cases, $\mathrm{Al}$ and $\mathrm{Si}$. Agreement for non-relativistic results (columns "NR") between VASP (optimal PAW data sets), WIEN2k, and best (aug-ccPCV5Z) DALTON shieldings is in general excellent. Specifically for Al, the maximum difference between non-relativistic "optimal" VASP and the best DALTON shieldings is just $0.8 \mathrm{ppm}$ (and the WIEN2k values are very close, as well).

For $\mathrm{Si}$, the maximum deviation between nonrelativistic "optimal" VASP and the best DALTON results increases to $4 \mathrm{ppm}$ for the strongly deshielded limit $\left(\mathrm{SiH}_{2}\right)$, which is still quite good. We observe a progressive increase of the difference between VASP and DALTON results with decreasing shielding. The nonrelativistic WIEN2k shieldings are 3 to $7 \mathrm{ppm}$ lower than the results obtained with VASP, and 2 to $3 \mathrm{ppm}$ lower than the best DALTON results.

In the scalar-relativistic case, inclusion of the small component $\left({ }^{+} B\right)$ has a noticeable effect (compare the column nine [VASP, optim, ${ }^{+} B$ ] and column ten [VASP, 
TABLE II. Calculated absolute isotropic chemical shieldings (in ppm). VASP calculations in were carried out in large boxes (see text). VASP "optim" results were obtained with Al_sv_nc, Si_sv_nc, P_sv_nc and F_h PAW data sets. "NR" denote non-relativistic calculations. " $B$ " denotes scalar relativistic calculations with two-component KS-orbitals in the atomic/PAW spheres. The DALTON calculations are non-relativistic using GIAOs and have uncontracted basis sets. Identical molecular geometries were used in the WIEN2k, VASP and DALTON calculations. Four slightly differently deformed tetrahedral geometries were used for $\left[\mathrm{AlH}_{4}\right]^{-}$. "MAD" denotes "mean absolute deviation" for the set of molecules that have WIEN2k results.

\begin{tabular}{|c|c|c|c|c|c|c|c|c|c|c|}
\hline & \multicolumn{5}{|c|}{ All-electron methods } & \multicolumn{5}{|c|}{ GIPAW } \\
\hline & \multicolumn{2}{|c|}{ WIEN2k } & \multicolumn{3}{|c|}{ DALTON2011, aug-cc-pCVXX } & \multicolumn{4}{|c|}{ VASP } & \multirow[t]{2}{*}{ other } \\
\hline & NR & ${ }^{+} B$ & $\begin{array}{c}5 Z \\
N R\end{array}$ & $\begin{array}{l}\text { QZ } \\
\text { NR }\end{array}$ & $\begin{array}{l}\mathrm{TZ} \\
\mathrm{NR} \\
\end{array}$ & $\begin{array}{c}\text { optim. } \\
\text { NR }\end{array}$ & $\begin{array}{c}\text { optim. } \\
+\end{array}$ & optim. & stand. & \\
\hline \multicolumn{11}{|c|}{ Al shieldings ${ }^{\mathrm{a}}$} \\
\hline \multirow{4}{*}[\mathrm{AlH}_{4}]{$^{-}$} & & & 477.94 & 478.23 & 480.89 & 477.50 & 476.08 & 477.89 & 483.93 & \\
\hline & & & 478.15 & 478.43 & 481.10 & 477.71 & 476.30 & 478.11 & 484.21 & \\
\hline & & & 478.25 & 478.53 & 481.20 & 477.84 & 476.42 & 478.23 & 484.31 & \\
\hline & & & 478.92 & 479.21 & 481.88 & 478.47 & 477.07 & 478.87 & 484.97 & \\
\hline $\mathrm{Al}_{2} \mathrm{H}_{6}$ & 402.8 & 401.2 & 404.25 & 404.49 & 406.96 & 404.60 & 402.30 & 404.56 & 411.13 & \\
\hline $\mathrm{AlH}_{3}$ & 249.7 & 245.9 & 250.24 & 250.46 & 254.22 & 251.03 & 246.84 & 250.06 & 258.54 & \\
\hline \multicolumn{11}{|c|}{ Si shieldings ${ }^{a}$} \\
\hline $\mathrm{Si}_{2} \mathrm{H}_{6}$ & 436.9 & 433.9 & 439.38 & 439.65 & 443.27 & 439.98 & 436.29 & 439.21 & 443.01 & \\
\hline $\mathrm{SiH}_{4}$ & 431.6 & 429.5 & 433.63 & 433.92 & 437.76 & 434.30 & 430.94 & 433.88 & 437.68 & \\
\hline $\mathrm{Si}_{2} \mathrm{H}_{4}$ & 235.9 & 230.2 & 238.28 & 238.53 & 241.53 & 239.55 & 233.01 & 237.38 & 240.56 & \\
\hline $\mathrm{SiH}_{2}$ & -533.4 & -545.4 & -530.20 & -530.34 & -527.12 & -526.34 & -538.36 & -528.67 & -523.91 & \\
\hline \multicolumn{6}{|c|}{$\mathrm{P}$ shieldings ${ }^{\mathrm{a}}$} & & & & & $\mathrm{QE}^{\mathrm{b}}$ \\
\hline $\mathrm{P}_{4}$ & 858.8 & 860.0 & 861.56 & 861.68 & 862.31 & 862.54 & 862.54 & 863.27 & 864.49 & 861.16 \\
\hline $\mathrm{PH}_{3}$ & & & 576.50 & 576.82 & 580.02 & 575.77 & 572.80 & 575.73 & 579.61 & 577.47 \\
\hline $\mathrm{P}_{2} \mathrm{H}_{4}$ & 517.0 & 513.0 & 520.11 & 520.42 & 523.61 & 519.46 & 514.79 & 518.16 & 522.93 & 519.68 \\
\hline $\mathrm{H}_{3} \mathrm{PO}_{4}$ & 281.8 & 275.1 & 284.65 & 285.00 & 289.31 & 283.87 & 276.30 & 281.21 & 292.51 & 285.73 \\
\hline $\mathrm{PF}_{3}$ & 153.9 & 145.7 & 155.72 & 155.94 & 159.74 & 156.05 & 146.87 & 152.94 & 156.58 & 158.02 \\
\hline $\mathrm{P}_{2}$ & -306.0 & -328.0 & -301.79 & -301.32 & -298.10 & -301.98 & -323.83 & -313.77 & -318.00 & -318.62 \\
\hline \multicolumn{6}{|c|}{ F shieldings ${ }^{c}$} & & & & & CASTEP $^{\mathrm{c}}$ \\
\hline $\mathrm{CH}_{3} \mathrm{~F}$ & & & 451.65 & 451.74 & 452.26 & 450.91 & 451.03 & 451.10 & 452.45 & 452.1 \\
\hline $\mathrm{HF}$ & 399.0 & 398.7 & 399.98 & 400.21 & 401.02 & 398.64 & 398.47 & 398.70 & 398.97 & 398.8 \\
\hline $\mathrm{C}_{6} \mathrm{~F}_{6}$ & & & 316.73 & 317.14 & 319.20 & 314.99 & 314.15 & 314.65 & 313.72 & 310.6 \\
\hline $\mathrm{CH}_{2} \mathrm{~F}_{2}$ & & & 301.56 & 301.98 & 304.13 & 299.98 & 298.69 & 299.24 & 297.81 & 298.7 \\
\hline $\mathrm{CF}_{4}$ & 211.2 & 209.2 & 212.35 & 212.94 & 216.14 & 210.51 & 208.67 & 209.51 & 205.01 & 207.0 \\
\hline $\mathrm{PF}_{3}$ & 176.8 & 177.7 & 178.20 & 178.90 & 183.41 & 173.76 & 173.08 & 174.25 & 172.08 & \\
\hline $\mathrm{CFCl}_{3}$ & & & 120.02 & 120.79 & 124.94 & 117.49 & 114.22 & 115.35 & 114.92 & 113.2 \\
\hline $\mathrm{NF}_{3}$ & -62.5 & -68.2 & -59.52 & -58.33 & -52.04 & -63.18 & -67.70 & -66.01 & -74.86 & -73.5 \\
\hline $\mathrm{F}_{2}$ & -293.4 & -299.8 & -288.21 & -286.56 & -276.99 & -292.54 & -298.33 & -295.93 & -307.14 & -296.3 \\
\hline MAD & 0.0 & & 2.4 & 2.8 & 6.6 & 2.5 & & & & \\
\hline MAD & 2.4 & & 0.00 & 0.45 & 4.15 & 1.63 & & & & \\
\hline MAD & & 0.0 & & & & & 2.1 & 5.4 & 9.3 & \\
\hline
\end{tabular}

${ }^{\text {a }}$ See supplementary material for molecular geometries.

${ }^{\text {b }}$ QE shieldings from Ref. 13 .

${ }^{c}$ Molecular geometries and CASTEP shieldings from Ref. 36 (except for $\mathrm{PF}_{3}$ ).

optim] in Table凹): in the high shielding limit, the shieldings differ by just 2-3 ppm, whereas in the low shielding limit of inclusion of the $B$-component reduces the shielding by $10 \mathrm{ppm}\left(\mathrm{SiH}_{2}\right)$. The relativistic corrections to the shielding, calculated as the difference between the twocomponent scalar-relativistic and non-relativistic shieldings, are quite similar for VASP and WIEN2k: they differ by $\sim 1 \mathrm{ppm}$. This supports the validity of our twocomponent implementation.

Phosphorous is a more critical case. Creating accu- rate PAW data sets for phosphorous, that include $2 s$ and $2 p$ states as valence orbitals, is challenging. The "optimal" $\mathrm{P}$ data set is created with $p$ core radii of 1.95 a.u., where the $2 p$ core orbitals have almost negligible ampli-

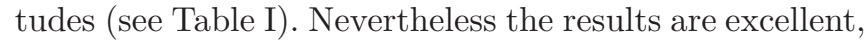
with maximal differences between VASP and DALTON of $1 \mathrm{ppm}$. Even for the difficult case of $\mathrm{P}_{2}$, which is strongly deshielded, the agreement is very good. The WIEN2k shieldings are 2-4 ppm lower than the corresponding VASP and DALTON results. 
Relativistic effects are increasingly more important towards the deshielded limit. For $\mathrm{P}_{4}$ relativistic effects amount to only a 0-1 ppm increase of the shielding, whereas for $\mathrm{P}_{2}$ the reduction is $22 \mathrm{ppm}$ (with VASP as well as with WIEN2k). Here again, inclusion of the small component $\left({ }^{+} B\right)$ has a significant effect. Neglecting explicit contributions from the small component of the scalar-relativistic orbitals yields an increase of the $\mathrm{P}_{2}$ shieldings of $10 \mathrm{ppm}$. Including the contributions of the small component, the VASP and WIEN2k relativistic corrections (difference between "NR" and "+ $B$ ") are identical to within $\sim 1 \mathrm{ppm}$.

The agreement between VASP ("optim.") and QE shieldings is good for high shieldings, but becomes worse towards the low shielding limit. We attribute this to the fact that in the QE calculations the $2 s$ and $2 p$ electrons were treated as part of the core. However, using the "standard" PAW data sets the VASP shieldings still significantly differ from the QE results. This illustrates to what extent the results depend on the particulars (matching radii, etc.) of the "standard" PAW data sets.

The last molecular test systems are fluorine compounds. Again agreement between VASP and DALTON is very good, but, as for the silicon compounds, discrepancies with increasing deshielding are observed. In general, VASP calculations tend to predict smaller shieldings (and stronger deshielding), with differences of up to $4 \mathrm{ppm}$ for $\mathrm{PF}_{3}, \mathrm{CFCl}_{3}, \mathrm{NF}_{3}$ and $\mathrm{F}_{2}$. Here, carefully scrutinizing the convergence of the DALTON calculations for $\mathrm{F}_{2}$ and $\mathrm{NF}_{3}$ shows that the basis set convergence using Gaussian basis sets is slow, and differences between QZ and $5 \mathrm{Z}$ can be as large as $1.5 \mathrm{ppm}\left(\mathrm{F}_{2}\right)$. In view of this it is not unlikely that the DALTON results might still be inaccurate for these strongly deshielded cases $\left(\mathrm{F}_{2}\right.$ and $\mathrm{NF}_{3}$ ), despite the use of uncontracted $5 \mathrm{Z}$ basis sets. It has been suggested that relaxation of the $\mathrm{F} 1 s$ core states might play a role (see Ref. 36 ), i.e., the frozen core approximation in the GIPAW calculations may also explain part of the difference between the VASP and DALTON results: the close agreement between VASP (frozen core) and WIEN2k (all-electron) results for $\mathrm{F}_{2}$ and $\mathrm{NF}_{3}$, however, does not support this. In fact, calculations with WIEN2k show the F $1 s$ contribution to be constant within the series (305.8/306.4 ppm for non-/scalarrelativistic calculations) In these calculations the F $1 s$ state self-consistently adapts to the spherical part of the potential. Admixture of $p$ and higher angular momentum states into the $\mathrm{F} 1 s$ is not possible, but this should not really matter for a localized 1s core state at $-48 \mathrm{Ry}$. The WIEN2k and VASP fluorine shieldings agree very well, with (NR) differences less than $1 \mathrm{ppm}$, except for $\mathrm{PF}_{3}$ where the (NR) difference is a bit larger (3 ppm). Finally, agreement with the CASTEP GIPAW results of Sadoc et al ${ }^{36}$ is quite good as well (our DALTON molecular quantum chemical shieldings are also very close to their Gaussian basis set results).

All in all, Table II shows that the "optimal" VASP potentials constitute a very stringent reference for fu- ture tests. The agreement between VASP shieldings and the Gaussian based DALTON calculations is excellent. The shieldings obtained with WIEN2k are generally a few ppm smaller than those obtained with VASP. This might relate to the numerically virtually exact basis sets close to the nucleus in the WIEN2k calculations. For strongly deshielded cases $\left(\mathrm{SiH}_{2}, \mathrm{P}_{2}, \mathrm{~F}_{2}\right)$ the deviations between DALTON, WIEN2k, and VASP calculations are larger (up to $7 \mathrm{ppm}$ ). For these three cases the WIEN2k results are always the most negative ones.

For heavier elements, the treatment of the so-called semi-core states, e.g., the low-lying $2 p$ states of $\mathrm{Si}$ or $\mathrm{P}$ becomes quite challenging. On the one hand, because of the very short bond-distances and anisotropic bonding situation in these molecules the splitting of the $2 p_{x}, 2 p_{y}$, and $2 p_{z}$ states may reach 10-25 mRy. On the other hand the spin-orbit splitting of these states is already more then twice as large. In the calculations presented above, spin-orbit coupling has been neglected. With WIEN2k it is possible to estimate the influence of SOC in the $2 p$ manifold on the NMR shieldings: it turns out to be a fairly small effect.

Scalar relativistic effects can be substantial as can be the contribution of the small-component, even for the light elements considered here. However, often they appear to increase (in size) with reduced shielding. So for calculating differences, which in practice is most important, we think they can often be safely neglected.

In general, we observe that it is more difficult to predict and converge shieldings in the deshielded limit. This is not unusual: in quantum chemical calculations, the diamagnetic contribution, which depends only on the charge density, converges rapidly. The paramagnetic contribution, which involves the Green's function and a sum over empty states, converges slower. For fluorine, the diamagnetic contribution is approximately $500 \mathrm{ppm}$ (DALTON number), and only weakly dependent on the molecular composition. The paramagnetic contribution goes from approximately $-50 \mathrm{ppm}$ for $\mathrm{CH}_{3} \mathrm{~F}$ in the shielded limit to approximately $-800 \mathrm{ppm}$ for $\mathrm{F}_{2}$ in the deshielded limit. The latter exhibits a substantial variation with basis set size, and the variation of these contributions with the basis set size is an order of magnitude larger than the variation of the diamagnetic contribution.

We finish with a final look at the difference between standard and optimal VASP PAW potentials. For fluorine the agreement is excellent (except for the difficult strongly deshielded cases). This is not unexpected, since the same number of electrons are treated as valence. For $\mathrm{Al}(\mathrm{Si})$, the "optimal" $\mathrm{PAW}$ data sets give $\sim 7(4) \mathrm{ppm}$ smaller shieldings than the standard PAW potentials. For $\mathrm{P}$ the optimal data sets give smaller shieldings in the high shielding limit, and higher shieldings in the deshielded limit. Since small systematic offsets matter little for comparison with experiment, standard potentials will often give the right trends. Indeed, for $\left[\mathrm{AlH}_{4}\right]^{-}$ small deformations of the poly-anion result in very similar changes in shielding for all PAW data sets and all 

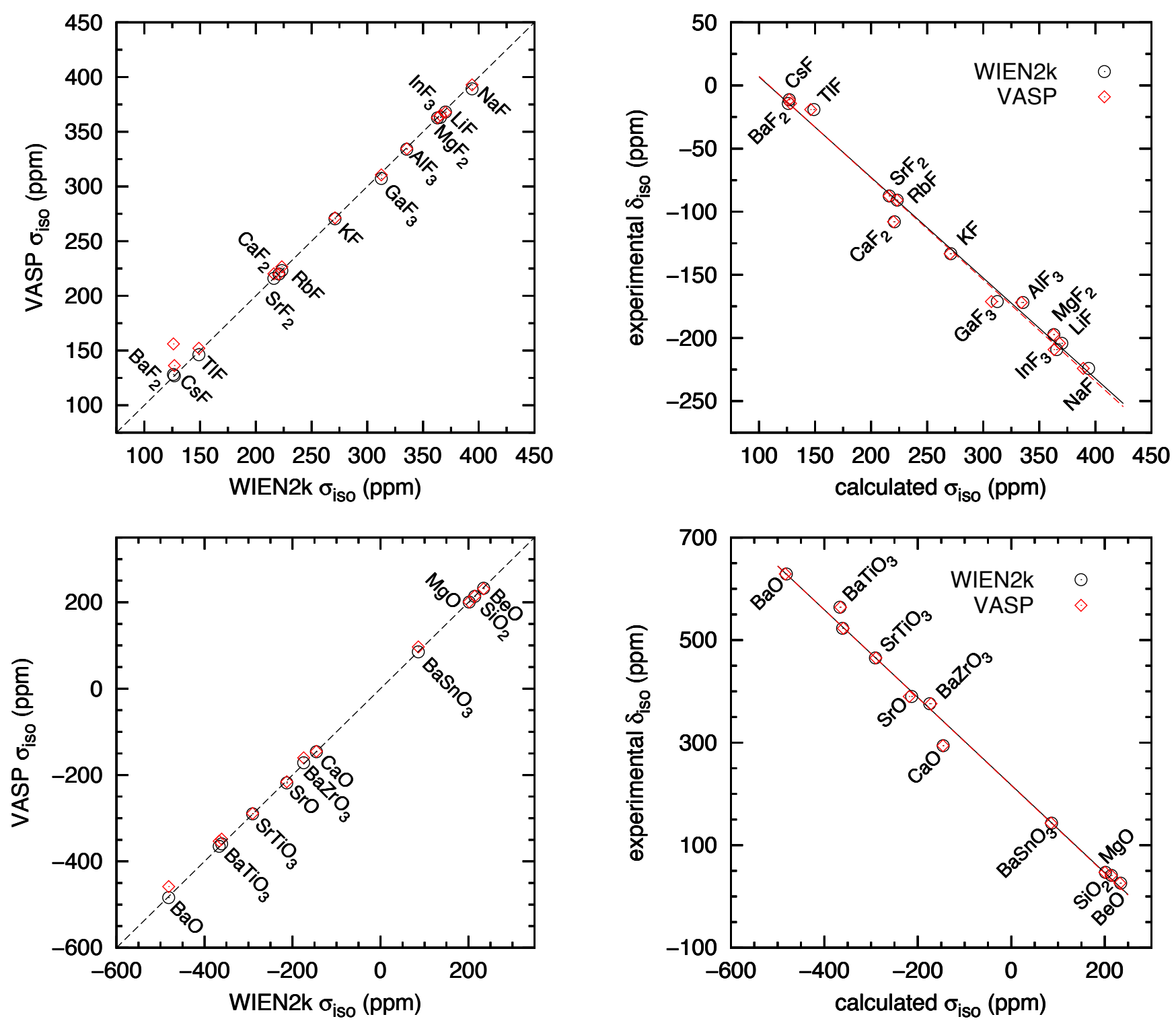

FIG. 2. Comparison of shieldings calculated with WIEN2k and VASP with optimal (black circles) and standard (red diamonds) PAW data sets.

quantum chemical basis sets. However, in critical cases we advise to check against the optimal potentials, because occasionally the trends can be broken. This is illustrated by $\mathrm{H}_{3} \mathrm{PO}_{4}$, where the standard data set gives an overestimation of the shielding by $\sim 10 \mathrm{ppm}$.

\section{B. Solids}

Table III lists the isotropic F and O NMR shieldings and magnetic susceptibilities for a range of fluoride and oxide systems, calculated using VASP and WIEN2k. As in the case of the molecular shieldings, using the high quality "optimal" PAW data sets, the VASP and

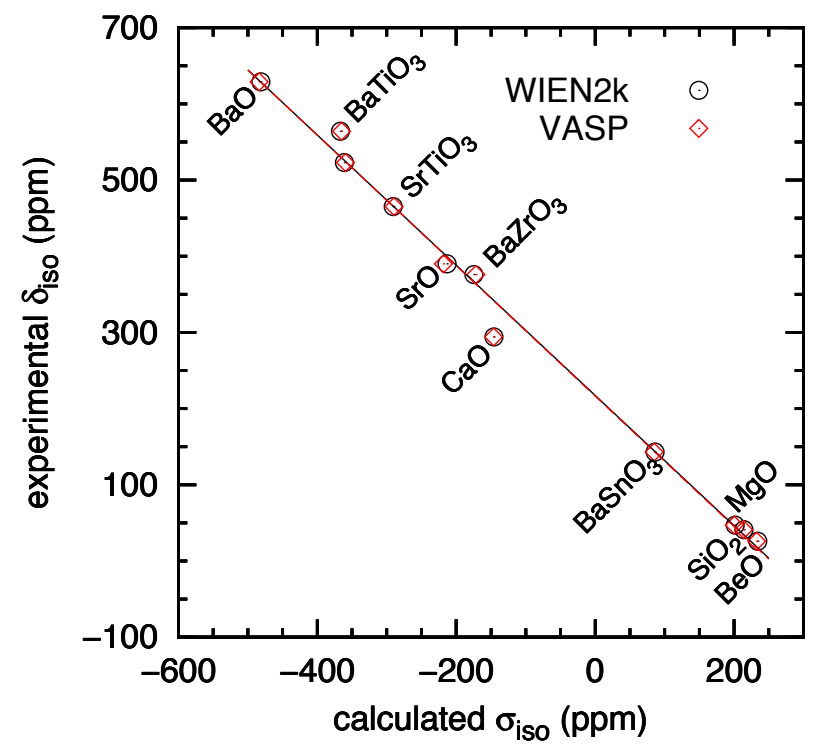

FIG. 3. Experimental shifts compared to calculated shieldings, for WIEN2k (black circles) and VASP (red diamonds). The lines are linear fits, with parameters according to Table IV]black solid line corresponds to WIEN2k, dashed red line corresponds to VASP.

WIEN2k results are in very good agreement. The largest deviations occur for $\mathrm{KF}, \mathrm{GaF}_{3}$ and $\mathrm{SrO}$. For $\mathrm{GaF}_{3}$ this can be traced back to a difference in calculated susceptibilities. Deviations are acceptable though, especially considering the shielding range of $\mathrm{F}$ and $\mathrm{O}$. This is illustrated in Fig. 2. by means of a plot of VASP shieldings versus shieldings obtained with WIEN2k.

Figure 2 furthermore shows that on the scale of the $\mathrm{F}$ and $\mathrm{O}$ shielding range in these compounds, using "standard" PAW data sets does not affect the results appreciably, except for the $\mathrm{Ba}$ and $\mathrm{Cs}$ compounds. For some of the other compounds there appear even to be slight 
TABLE III. Calculated isotropic $\mathrm{F}$ and $\mathrm{O}$ NMR shieldings $\sigma_{\text {iso }}$ (in ppm), calculated magnetic susceptibilities $\chi_{\mathrm{m}}$ (in $10^{-6} \mathrm{~cm}^{3} \mathrm{~mol}^{-1}$ ) and experimental chemical shifts $\delta_{\text {iso }}$ (in ppm). GIPAW $\mathrm{F}$ and $\mathrm{O}$ shieldings calculated with other codes (column "other GIPAW") were taken from Ref. 36 and Refs. 37 and 38, respectively. Susceptibilities are per mole f.u. Experimental susceptibilities are from Refs. 39 42. Structures are from Ref. 36 and the inorganic crystal structure database. ${ }^{11,43}$ All calculations are scalar relativistic with, inside the atomic spheres, one (VASP) and two (WIEN2k) component KS orbitals. VASP numbers calculated with $900 \mathrm{eV}(700 \mathrm{eV})$ kinetic energy cutoff in column optim. (standard). "MAD" denotes "mean absolute deviation".

\begin{tabular}{|c|c|c|c|c|c|c|c|c|c|}
\hline & \multicolumn{4}{|c|}{$\sigma_{\text {iso }}$} & \multirow{3}{*}{$\begin{array}{c}\delta_{\text {iso }} \\
\text { expt. }\end{array}$} & \multicolumn{4}{|c|}{$\chi_{\mathrm{m}}$} \\
\hline & \multirow{2}{*}{ WIEN2k } & \multicolumn{2}{|c|}{ VASP } & \multirow{2}{*}{$\begin{array}{c}\text { other } \\
\text { GIPAW }\end{array}$} & & \multirow{2}{*}{ WIEN2k } & \multicolumn{2}{|c|}{ VASP } & \multirow{2}{*}{ expt } \\
\hline & & optim. & standard & & & & optim. & standard & \\
\hline \multicolumn{10}{|c|}{ F shieldings } \\
\hline $\mathrm{NaF}$ & 393.98 & 389.21 & 392.86 & $395.8^{36}$ & $-224.2^{36}$ & -16.0 & -10.9 & -11.6 & -15.6 \\
\hline $\mathrm{LiF}$ & 370.12 & 368.14 & 367.65 & $369.3^{36}$ & $-204.3^{36}$ & -10.8 & -9.1 & -7.7 & -10.1 \\
\hline $\mathrm{InF}_{3}$ & 365.55 & 363.17 & 365.02 & & $-209.2^{44}$ & -54.8 & -43.4 & -35.7 & \\
\hline $\mathrm{MgF}_{2}$ & 362.93 & 362.59 & 362.92 & $362.7^{36}$ & $-197.3^{36}$ & -23.5 & -24.1 & -38.8 & -22.7 \\
\hline$\alpha-\mathrm{AlF}_{3}$ & 335.32 & 334.06 & 334.51 & & $-172.0^{45}$ & -30.1 & -28.3 & -24.9 & -13.9 \\
\hline $\mathrm{GaF}_{3}$ & 312.51 & 307.23 & 310.63 & & $-171.2^{44}$ & -42.6 & -29.5 & -28.2 & \\
\hline $\mathrm{KF}$ & 271.08 & 270.41 & 271.27 & $268.1^{36}$ & $-133.3^{36}$ & -23.4 & -25.1 & -23.2 & -23.6 \\
\hline $\mathrm{RbF}$ & 223.34 & 223.07 & 226.54 & $221.3^{36}$ & $-90.9^{36}$ & -31.6 & -31.0 & -34.0 & -31.9 \\
\hline $\mathrm{CaF}_{2}$ & 220.72 & 219.99 & 220.02 & $220.0^{36}$ & $-108.0^{36}$ & -25.8 & -26.7 & -22.7 & -28 \\
\hline $\mathrm{SrF}_{2}$ & 216.17 & 216.05 & 220.17 & $215.3^{36}$ & $-87.5^{\frac{36}{1}}$ & -34.4 & -33.6 & -34.6 & -37.2 \\
\hline $\mathrm{TlF}$ & 148.92 & 146.10 & 152.06 & & $-19.1^{46}$ & -50.7 & -30.6 & -42.8 & -44.4 \\
\hline $\mathrm{CsF}$ & 127.01 & 126.94 & 136.24 & $136.3^{36}$ & $-11.2^{36}$ & -44.3 & -40.9 & -44.9 & -44.5 \\
\hline $\mathrm{BaF}_{2}$ & 126.05 & 128.19 & 156.10 & $151.9^{36}$ & $-14.3^{36}$ & -44.8 & -42.2 & -51.7 & -51 \\
\hline $\operatorname{MAD}(\mathrm{F})$ & 0.00 & 1.76 & 4.41 & 5.0 & & 0.0 & 4.9 & 6.4 & \\
\hline \multicolumn{10}{|c|}{ O shieldings } \\
\hline $\mathrm{BeO}$ & 234.17 & 232.59 & 231.44 & $232.2^{37}$ & $26 .^{47}$ & -12.6 & -11.2 & -10.8 & -11.9 \\
\hline $\mathrm{SiO}_{2}$ & 214.21 & 213.83 & 213.95 & & 41.48 & -24.3 & -24.9 & -23.7 & -28.6 \\
\hline $\mathrm{MgO}$ & 201.77 & 200.25 & 200.82 & $198.0^{37}$ & $47 \cdot \frac{47}{.}$ & -15.8 & -18.3 & -15.8 & -10.2 \\
\hline $\mathrm{BaSnO}_{3}$ & 86.08 & 85.09 & 96.61 & $98.0^{37}$ & 143.37 & -73.1 & -61.5 & -70.4 & \\
\hline $\mathrm{CaO}$ & -145.56 & -146.05 & -145.30 & $-156.6^{38}$ & $294^{38}$ & -11.4 & -13.4 & -15.7 & -15.0 \\
\hline $\mathrm{BaZrO}_{3}$ & -174.74 & -171.75 & -160.04 & $-172.8^{37}$ & 376.49 & -39.3 & -39.6 & -62.9 & \\
\hline $\mathrm{SrO}$ & -213.16 & -218.29 & -215.53 & $-205.2^{37}$ & 390.47 & -16.5 & -17.6 & -22.4 & -35 \\
\hline $\mathrm{SrTiO}_{3}$ & -290.61 & -289.75 & -289.14 & $-287.3^{37}$ & $465 \stackrel{49}{\frac{20}{4}}$ & -10.0 & -9.8 & -36.5 & -18.6 \\
\hline $\mathrm{BaTiO}_{3}$ & -361.06 & -359.49 & -348.40 & $-347.4^{37}$ & $5233^{37}$ & -12.4 & -11.0 & -48.4 & \\
\hline $\mathrm{DadiU}_{3}$ & -366.80 & -365.20 & -353.43 & $-357.9^{37}$ & $564 !^{37}$ & & & & \\
\hline $\mathrm{BaO}$ & -481.43 & -483.71 & -458.46 & $-444.3^{37}$ & 629.47 & -17.3 & -18.4 & -30.7 & -29.1 \\
\hline $\operatorname{MAD}(\mathrm{O})$ & 0.00 & 1.76 & 7.48 & 10.2 & & 0.0 & 2.2 & 11.5 & \\
\hline
\end{tabular}

improvements (e.g. for NaF). All Cs and Ba data sets have unfrozen $5 s$ and $5 p$ semi-core states, i.e. the shallow core states are allowed to be polarized in the crystal field. This, apparently, is not sufficient to get accurate shieldings. The standard $\mathrm{Ba}$ data set has $\mathrm{PAW}$ matching radii of $2.8(2.7)$ Bohr for the $s(p)$ channel, with 2 projectors per channel. The "optimal" set has much smaller radii of 1.3-1.4 (2.2), and norm-conserving pseudo partial waves for the $p$ channel. Evidently this substantially reduces inaccuracies due to incompleteness in the PAW sphere, which results in more accurate current densities that are "felt" in the induced field at the neighboring nuclei. With the "optimal" data sets, agreement with WIEN2k is very good.

In general the magnetic susceptibilities obtained with VASP using the "optimal" PAW data sets are in fair agreement with the susceptibilities obtained with WIEN2k, with some exceptions for compounds with heavier nuclei (TIF being the most dramatic). The susceptibilities calculated using the "standard" PAW data sets in many cases are in quite poor agreement with the all-electron WIEN2k results (e.g. $\mathrm{SrTiO}_{3}$ and $\mathrm{BaZrO}_{3}$ ). This makes sense, as the YPM expression for the magnetic susceptibility lacks one-centre corrections (Eqs. 47 and 48 of Ref. 4), and is expected to become more accurate when PAW data sets become harder (smaller core radii and/or norm-conserving). Shieldings calculated discarding the susceptibility contribution of Eq.12]are listed as supplementary material.

In Fig. 3 we compare WIEN2k and VASP shieldings ("optimal" PAW data sets) to chemical shifts from experiment. A fit is done, according to:

$$
\delta_{\text {iso }}^{\text {exp }}=\sigma_{\text {ref }}-m \sigma_{\text {iso }}^{\text {calc }} .
$$

Fit results are in Table IV] Note that the Ca compounds are well besides the fitted straight lines. Indeed, it is 
TABLE IV. Fit parameters according to Eq. 35 with standard errors in brackets and Pearson correlation coefficient $r$.

\begin{tabular}{lrrr}
\hline \hline & \multicolumn{1}{c}{$\sigma_{\text {ref }}$} & $m$ & $r$ \\
\hline fluorides & & & \\
WIEN2k & $86.47(7.08)$ & $-0.7964(0.0250)$ & -0.9946 \\
VASP (optim) & $87.76(7.22)$ & $-0.8056(0.0257)$ & -0.9945 \\
VASP (standard) & $101.64(9.54)$ & $-0.8429(0.0337)$ & -0.9914 \\
oxides & & & \\
WIEN2k & $217.23(6.77)$ & $-0.8546(0.0247)$ & -0.9963 \\
VASP (optim) & $216.67(7.00)$ & $-0.8558(0.0256)$ & -0.9960 \\
VASP (standard) & $220.65(7.79)$ & $-0.8724(0.0292)$ & -0.9950 \\
\hline \hline
\end{tabular}

known that the empty $\mathrm{Ca} 3 d$-states are too close to the valence band maximum in DFT, resulting in a deviation of the O shift. 38

The fit of Eq. 35 relates the calculated shieldings to chemical shifts from experiment. Table IV shows that the slope $m$ amd reference shielding $\sigma_{\text {ref }}$ from VASP calculations with optimal PAW data sets and as obtained with WIEN2k are in excellent agreement.

\section{CONCLUSIONS}

In the calculation of NMR shieldings for the molecular systems considered in this paper we have pushed the convergence of the results of the DALTON (Gaussian basis set), WIEN2k (APW+lo), and VASP (GIPAW) calculations with respect to their basis sets as far as practicably possible. If this is done, generally excellent agreement can be obtained for very different codes and implementations. Specifically, for Si, Al and P agreement of GIPAW calculations (VASP) with the all-electron Gaussian basis set results (DALTON) is excellent, with inaccuracies of several ppm in the extreme deshielded limit for $\mathrm{SiH}_{2}$. For $\mathrm{F}$, in the deshielded range, differences of up to $4 \mathrm{ppm}$ occur, but in this case the DALTON calculations are probably still not fully converged with respect to the basis set size, and the VASP results are validated by the comparison to WIEN2k. Generally, the all-electron APW+lo WIEN2k shieldings agree very well with those obtained from DALTON and VASP calculations, although the WIEN2k shieldings are consistently slightly lower than the DALTON (and most of the VASP) results. This might be due to the superior quality of the APW+lo basis sets of WIEN2k close to the nuclei.

Scalar relativistic effects, even for light nuclei, can be substantial. They increase towards the deshielded limit, where inclusion of the small-component of the wave function can considerably affect the shielding.

In general, the agreement between VASP and WIEN2k shieldings is very good for the molecular and the solid state systems considered here. We consider this to be a validation of our norm-conserving and hard GW PAW data sets. Completeness of the projector functions/partial waves inside the PAW spheres is crucially important, not only for the elements for which the shielding is calculated, but for other atomic constituents as well: This is especially true for $\mathrm{Cs}$ and $\mathrm{Ba}$, that have very shallow semi-core states. Large matching radii in the pseudization of $\mathrm{Cs}$ and $\mathrm{Ba}$ (as regularly used) yield wrong oxygen and fluorine shifts. Reducing these radii and the use of norm-conserving partial waves yields a more accurate description of the current density already on the plane wave grid, i.e., the results are less affected by undercompleteness of the PAW one-center basis. This, however, generally comes at the price of an increase in the cutoff energy of the plane wave basis set. Standard VASP PAW data sets, although being less accurate, describe trends and chemical differences quite well. Hence, they can be used in shielding calculations, although for small differences double checks with more accurate PAW data sets are in order. In general, it is more difficult to predict and converge shieldings in the deshielded limit, where the paramagnetic contribution, that involves the Green's function, is larger.

Harder data sets give, in general, better values for the susceptibilities, since one-centre corrections are presently missing for the susceptibilities. In most cases reasonable susceptibilities can often be obtained with standard data sets already, and the contribution of the susceptibility to the shielding is, in general, modest anyway. This is crucial, since our, as well as other implementations use only one-centre corrections for the current density.

\section{SUPPLEMENTARY MATERIAL}

See supplementary material for the structure of the Al, $\mathrm{Si}$ and $\mathrm{P}$ containing molecules and solid state shieldings calculated without the $G=0$ contribution.

\section{ACKNOWLEDGMENTS}

We thank Dr. F. M. Vasconcelos for useful discussions. The work was partly supported by the Austrian Science Fund (FWF) within the Spezialforschungsbereich Vienna Computational Materials Laboratory (SFB ViCoM, F41). The work of GAW is part of the research programme of the "Stichting voor Fundamenteel Onderzoek der Materie (FOM)", which is financially supported by the "Nederlandse Organisatie voor Wetenschappelijk Onderzoek (NWO)".

${ }^{1}$ T. Helgaker, M. Jaszuński, and K. Ruud, Chem. Rev. 99, 293 (1999).

${ }^{2}$ F. Mauri, B. G. Pfrommer, and S. G. Louie, Phys. Rev. Lett. 77, 5300 (1996).

${ }^{3}$ C. J. Pickard and F. Mauri, Phys. Rev. B 63, 245101 (2001).

${ }^{4}$ J. R. Yates, C. J. Pickard and F. Mauri, Phys. Rev. B 76, 024401

(2007).

${ }^{5}$ P. E. Blöchl, Phys. Rev. B 50, 17953 (1994).

${ }^{6}$ R. Ditchfield, Mol. Phys. 27, 789 (1974). 
${ }^{7}$ S. J. Clark, M. D. Segall, C. J. Pickard, P. J. Hasnip, M. J. Probert, K. Refson and M. C. Payne, Z. Kristallogr. 220, 567 (2005).

${ }^{8}$ Paolo Giannozzi, Stefano Baroni, Nicola Bonini, Matteo Calandra, Roberto Car, Carlo Cavazzoni, Davide Ceresoli, Guido L Chiarotti, Matteo Cococcioni, Ismaila Dabo, Andrea Dal Corso, Stefano de Gironcoli, Stefano Fabris, Guido Fratesi, Ralph Gebauer, Uwe Gerstmann, Christos Gougoussis, Anton Kokalj, Michele Lazzeri, Layla Martin-Samos, Nicola Marzari, Francesco Mauri, Riccardo Mazzarello, Stefano Paolini, Alfredo Pasquarello, Lorenzo Paulatto, Carlo Sbraccia, Sandro Scandolo, Gabriele Sclauzero, Ari P Seitsonen, Alexander Smogunov, Paolo Umari, and Renata M Wentzcovitch, J. Phys.: Condens. Matter 21, 395502 (2009), http://www.quantum-espresso.org

${ }^{9}$ T. Charpentier, Solid State Nucl. Magn. Reson. 40, 1 (2011).

${ }^{10}$ R. Laskowski and P. Blaha, Phys. Rev. B 85, 035132 (2012).

${ }^{11}$ R. Laskowski, P. Blaha, and F. Tran, Phys. Rev. B 87, 195130 (2013).

${ }^{12}$ R. Laskowski and P. Blaha, Phys. Rev. B 89, 014402 (2014).

${ }^{13}$ F. Vasconcelos, G. A. de Wijs, R. W. A. Havenith, M. Marsman, and G. Kresse, J. Chem. Phys. 139, 014109 (2013).

${ }^{14}$ Dalton, a molecular electronic structure program, Release Dalton2011 (2011), see http://daltonprogram.org

${ }^{15}$ F. Mauri and S. G. Louie, Phys. Rev. Lett. 76, 4246 (1996).

${ }^{16}$ A. M. Rappe, K. M. Rabe, E. Kaxiras, and J. D. Jaonnopoulos, Phys. Rev. B 41, 1227 (1990).

${ }^{17}$ G. Kresse and J. Hafner, J. Phys.: Condens. Matter 6, 8245 (1994).

${ }^{18}$ G. Kresse and D. Joubert, Phys. Rev. B 59, 1758 (1999).

${ }^{19}$ T. Gregor, F. Mauri, and R. Car, J. Chem. Phys. 111, 1815 (1999).

${ }^{20}$ J. P. Perdew, K. Burke, and M. Ernzerhof, Phys. Rev. Lett. 77, 3865 (1996).

${ }^{21}$ J. P. Perdew, K. Burke, and M. Ernzerhof, Phys. Rev. Lett. 78, 1396 (1997).

${ }^{22}$ G. Kresse, M. Marsman, and J. Furthmüller, VASP the Guide (Vienna, March 26, 2015), http://cms.mpi.univie.ac.at/vasp/vasp/vasp.html

${ }^{23}$ J. Klimeš, M. Kaltak, and G. Kresse, Phys. Rev. B 90, 075125 (2014).

${ }^{24}$ E. van Lenthe, E. J. Baerends, and J. G. Snijders, J. Chem. Phys. 99, 4597 (1993).

${ }^{25}$ T. Helgaker, P. J. Wilson, R. D. Amos, and N. C. Handy, J. Chem. Phys. 113, 2983 (2000).

${ }^{26}$ T. H. Dunning, Jr., J. Chem. Phys. 90, 1007 (1989).
${ }^{27}$ D .E. Woon, and T. H. Dunning, Jr., (to be published).

${ }^{28}$ D .E. Woon, and T. H. Dunning, Jr., J. Chem. Phys. 98, 1358 (1993).

${ }^{29}$ D .E. Woon, and T. H. Dunning, Jr., J. Chem. Phys. 103, 4572 (1995).

${ }^{30}$ K. A. Peterson, and T. H. Dunning, Jr., J. Chem. Phys. 117, 10548 (2002).

${ }^{31}$ R. A. Kendall, T. H. Dunning, Jr. and R. J. Harrison, J. Chem. Phys. 96, 6796 (1992).

${ }^{32}$ D. Feller, (unpublished).

${ }^{33}$ S. Mielke, (unpublished).

${ }^{34}$ D. Feller, J. Comp. Chem. 17, 1571 (1996).

${ }^{35}$ K. L. Schuchardt, B. T. Didier, T. Elsethagen, L. Sun, V. Gurumoorthi, J. Chase, J. Li, and T. L. Windus, J. Chem. Inf. Model. 47, 1045 (2007).

${ }^{36}$ A. Sadoc, M. Body, C. Legein, M. Biswal, F. Fayon, X. Rocquefelte, and F. Boucher, Phys. Chem. Chem. Phys. 13, 18539 (2011).

${ }^{37}$ D. S. Middlemiss, F. Blanc, C. J. Pickard, and C. P. Grey, J. Magn. Reson. 204, 1 (2010).

${ }^{38}$ M. Profeta, M. Benoit, F. Mauri, and C. J. Pickard, J. Am. Chem. Soc. 126, 12628 (2004).

${ }^{39}$ Landolt-Börnstein, Numerical Data and Functional Relationships in Science and Technology, New Series, II/16, Diamagnetic Susceptibility (Springer, Heidelberg, 1986).

${ }^{40}$ Landolt-Börnstein, Numerical Data and Functional Relationships in Science and Technology, New Series, II/2, II/8, II/10, II/11, II/12a, Coordination and Organometallic Transition Metal Compounds (Springer, Heidelberg, 1986).

${ }^{41}$ Tables de Constantes et Données Numérique, Volume 7, Relaxation Paramagnétique (Masson, Paris, 1957).

${ }^{42}$ H. P. R. Frederikse, and G. A. Candela, Phys. Rev. 147, 583 (1966).

43 http://icsd.fiz-karlsruhe.de/icsd

${ }^{44}$ B. Bureau, G. Silly, J. Buzaré, and J. Emery, Chem. Phys. 249, 89 (1999).

${ }^{45}$ P. J. Chupas, M. F. Ciraolo, J. C. Hanson, and C. P. Grey, J. Am. Chem. Soc. 123, 1694 (2001).

${ }^{46}$ S. Gabuda, S. Kozlova, and R. Davidovich, Chem. Phys. Lett. 263, 263 (1996).

${ }^{47}$ G. L. Turner, S. E. Chung, and E. Oldfield, J. Magn. Reson. 64, 316 (1985).

${ }^{48}$ M. Profeta, F. Mauri, and C. J. Pickard, J. Am. Chem. Soc. 125, 541 (2003).

${ }^{49}$ T. J. Bastow, P. J. Dirken, M. E. Smith, and H. J. Whitfield, J. Phys. Chem. 100, 18539 (1996). 